\title{
Vererbung und ihre Verweigerung: Eine Form des Romans im 19. Jahrhundert
}

"Ich selbst aber kann nicht weiterleben, da ich ja nicht gelebt habe, ich bin Lehm geblieben, den Funken habe ich nicht zum Feuer gemacht, sondern nur zur Illuminierung meines Leichnams benutzt."

(Franz Kafka an Max Brod, 5.7.1922)

Das 19. Jahrhundert ist in wissensgeschichtlicher Hinsicht von einem Begriff der Vererbung gekennzeichnet, in dem juristische und biologische Diskurse in einem wechselseitig befruchtenden Verhältnis stehen: Denn Vererbung bzw. heredity ist als Metapher aus dem juristischen in den biologischen Diskurs hineingewandert, ${ }^{1}$ während umgekehrt Vorstellungen aus dem Recht ihre Vor-Bilder in Naturgeschichte, -philosophie oder -wissenschaft finden. Das in diesem Feld zur Debatte stehende Wissen über Erbe und Vererbung lässt sich also nicht exklusiv einem einzigen Diskurs, einer einzelnen Disziplin und Methode respektive einem Sachgebiet und seinen Praktiken zuordnen, sondern erweist sich als ein durch Transfers und Transformationen zwischen Diskursen, Disziplinen, Methoden, Sachgebieten und Praktiken konstituiertes und wandelbares Wissen.

Auf dem Feld der Literatur lässt sich dieses komplexe Szenario gut beobachten - nicht nur, weil es prinzipiell kein Thema gibt, das nicht zum Gegenstand des Erzählens werden kann, oder weil die Literatur den produktionsästhetischen Imperativen des Schreibens (sich nicht zu wiederholen) oder den rezeptionsästhetischen Imperativen des Marktes (nicht zu langweilen) ausgesetzt ist. Vielmehr gibt es ein genuin literarisches vitales Interesse am Vererbungswissen des 19. Jahrhunderts, wie umgekehrt literarische Modelle des Erzählens, implizit oder explizit, eine wichtige Referenz zur Organisation des wissenschaftlichen Wissens von der Vererbung darstellen - bis zum Ende des Jahrhunderts. Denn auch wenn es um 1900 in den Naturwissen-

1 Vgl.: »heredity, formerly a concept restricted to the realm of law, began to be applied as a metaphor in matters of organic reproduction and successively became a concept of central importance to the life and human sciences.« Hans-Jörg Rheinberger/Staffan Müller-Wille: Heredity - The Formation of an Epistemic Space, in: Dies. (Hg.): Heredity Produced. At the Crossroads of Biology, Politics, and Culture, 1500-1870, Cambridge (MA) 2007, S. 3-34, hier S. 8. 
schaften noch »kein konsistentes und konsensfähiges Vererbungswissen ${ }^{2}$ gab, so gewinnt dieses doch rasch an Komplexität, so dass die Literatur zu Beginn des 20. Jahrhunderts ihren selbstbewussten Einflussanspruch auf die Life Sciences nicht länger vertritt. Dies hat Peter Morton anhand von Samuel Butlers Roman The Way of All Flesh und dem zeitlichen Auseinandertreten zwischen dessen Niederschrift (1873-1884) und der (auf Familienrücksichten zurückgeführten) verspäteten Publikation aus dem Nachlass 1903 konstatiert. $^{3}$

In seinem Roman erzählt Samuel Butler einen über drei Generationen sich erstreckenden Kampf der Väter gegen die Söhne. In diesem Kampf wird die Enterbung der Söhne als väterliche Waffe eingesetzt; so heißt es über den Vater: "Im Geiste malte er sich aus, wie er bald den einen, bald den anderen enterbte und mit seinem Geld Armenhäuser stiftete, bis er sich schließlich gezwungen sah, sie wieder als Erben einzusetzen, damit er beim nächsten Wutanfall das Vergnügen haben konnte, sie von neuem zu enterben. « ${ }^{4}$ Gegen diese andauernde Drohung entwirft der Erzähler Wunschphantasien, die zum einen rechtlicher Art sind. So imaginiert er eine neue Gesetzgebung, die all jenen, die ihre Nachkommen qua Testament unter Druck setzen, »für drei Monate die Testierfähigkeit entzieht«. ${ }^{5}$ Zum anderen imaginiert er eine neue Naturgesetzgebung, die die gleichzeitige Existenz von Eltern und Kindern überhaupt abschafft:

Warum müssen denn die Generationen einander überschneiden? Warum können wir nicht als Eier in hübschen kleinen Zellen, eingewickelt in zehn- bis zwanzigtausend Pfundnoten der Bank von England, in die Erde vergraben werden und dann

2 »Die [...] Faszination für Fragen der Vererbung darf dabei nicht darüber hinwegtäuschen, dass es innerhalb der Naturwissenschaften kein konsistentes und konsensfähiges Vererbungswissen gab. [...] Die Romane übersetzten nicht etwa ein naturwissenschaftliches Wissen in Literatur, sie waren selbst Teil der Produktion einer neuen Selbstverständlichkeit.«Christina Benninghaus: Brennende Sehnsüchte, heimliche Ängste. Kinderlosigkeit, Vererbung und Adoption im naturalistischen Roman um 1900, in: zeitenblicke 7, Nr. 3 (2008), URL: http://www.zeitenblicke.de/2008/3/benninghaus/index_html (letzter Zugriff: 1.9.2016)

3 "About this time [1903] [...] the increased complexity of biological research at last dissolved the very strong sentiment that the life sciences were too important to be left to the professionals. Butler, during the years that he wrote The Way of All Flesh (1873-84), really believed, and with some substance, that what he had to say could materially effect the direction of biological inquiry. But after 1903 the only literary figure still engaging in controversy with this supposition in mind was Bernard Shaw [...]. Peter Morton: The Vital Science. Biology and the Literary Imagination, 1860-1900, London 1984, S. 14.

4 Samuel Butler: Der Weg allen Fleisches, übers. von Helmut Findeisen, Berlin 1960, S. 32.

5 Ebd. 
beim Aufwachen feststellen, wie es bei der Schlupfwespe der Fall ist, daß Papa und Mama uns nicht nur einen reichlichen Vorrat bereitgestellt haben, sondern einige Wochen, bevor wir begannen, bewußt selbständig zu leben, von Spatzen verspeist worden sind? ${ }^{6}$

Diese radikale Unterbindung des direkten Austauschs zwischen den Generationen zielt auf einen konfliktfreien Raum jenseits der lebenden Individuen: Nur kollektiv verantwortete (erb-)rechtliche Regelungen und anonyme biologische Prozesse würden die Eltern mit ihren Kindern verbinden; jegliche direkte soziale Interaktion und psychologische Bindung wäre aufgehoben.

Wie aber wären daraus Romane zu machen? Butlers Roman selbst lebt ja von der generationellen Abfolge, dem genealogischen Rhythmus über drei Generationen, der zugleich einen Rhythmus der fortlaufenden patriarchalen Gewalt der Väter gegen die Söhne darstellt (vererben/enterben, Sohn sein/Vater sein). Damit entspricht er einem im 19. Jahrhundert verbreiteten Vererbungs- und Erzählmuster, das Patricia Tobin als Genealogical Imperative des Romans bezeichnet hat ${ }^{7}$ und das im Folgenden im Zentrum der Überlegungen zu einer Reihe von Romanen stehen soll. So geht es im ersten Teil um die Frage, welche literarischen Darstellungsformen der Begriff der Vererbung im 19. Jahrhundert privilegiert. Dabei soll auch die Organisation mehrgenerationaler Narrative interessieren, d.h. die erzählerische Proliferation etwa in Romanzyklen nach dem Prinzip des genealogischen Imperativs. Im zweiten Teil wird die Negation dieses Imperativs erörtert. Damit rückt jene vehemente Verweigerung von Fortpllanzung, Vererbung, Geschlechtlichkeit und Zukunft in den Fokus, die literarisch so außerordentlich produktiv geworden ist. Diese paradox anmutende Konstellation steht, wie zu zeigen ist, nicht nur im Zeichen der am Ende des 19. Jahrhunderts so prominenten Denkfigur der Degeneration, sondern arbeitet vor allem an der ästhetischen Modernisierung des Romans.

\section{Vererbungs- und Erzählmuster: Der genealogische Imperativ}

Im 19. Jahrhundert sind nicht nur die bürgerliche Familie und der zugehörige Roman in generativer und struktureller Hinsicht miteinander assoziiert, wie u.a. Tony Tanner gezeigt hat: »the genesis of both the modern family

6 Ebd., S. 93f.

7 Vgl. Patricia Tobin: Time and the Novel. The Genealogical Imperative, Princeton 1978. 
and what we call the novel - and their fates - are closely interlinked «. ${ }^{8}$ Auch Genealogie und Romanform sind engstens verknüpft, insofern das lineare Erzählen im epischen Roman mit der Idee von Vorfahren und Nachkommen sowie dem Denken in Ursprüngen und Abläufen verbunden ist. Patricia Tobin spricht diesbezüglich von "the homologous congruity between time-line, family-line and story-line« und erläutert diese Homologie: »events in time come to be perceived as begetting other events within a line of causality similar to the line of generations, with the prior event earning a special prestige as it is seen to originate, control, and predict future events. ${ }^{9}$

Eine solche Arbeit am Zusammenhang seitens des Romans richtet sich gegen die Kontingenz und Absichtslosigkeit lebendiger Prozesse ${ }^{10}$ und zielt auf eine dezidierte Sinnstiftung, die aus hierarchisierten Ursache-WirkungRelationen resultiert: »the prestige of cause over effect, in historical time, is analogous to the prestige of the father over the son ${ }^{11}{ }^{11}$ Die Geltungs- und Machtansprüche der Ursache über die Wirkung entsprechen jenen des Vaters über den Sohn - und verweisen so auf Familien- und Generationenromane als ein im 19. Jahrhundert enorm produktives, Sujet und Romanform verknüpfendes Genre.

Epische Romane versuchen demnach - analog zu Evolutionstheorie und Historismus -, im Sinne der zeitgenössisch vorherrschenden "GeneaLogik « ${ }^{12}$ die Vorgeschichten, Ursprünge und Herkünfte gegenwärtiger Verhältnisse zu erhellen und qua Entwicklungsdenken daraus resultierende $\mathrm{Zu}$ kunftsszenarien der Nachkommenschaften - ob im Modus des Fortschritts

8 Tony Tanner: Adultery in the Novel. Contract and Transgression, Baltimore 1979, S. 368. Christopher Flint postuliert auch für die britischen Romane des 18. Jahrhunderts eine "correspondance between eighteenth century fiction's depiction of the family and its mode of storytelling" und spricht in diesem Zusammenhang von »the urge to align the history of the family with the history of prose fiction". Christopher Flint: Family Fictions. Narrative and Domestic Relations in Britain, 1688-1798, Stanford 1998, S. 3; vgl. auch Denis Jonnes: The Matrix of Narrative. Family Systems and the Semiotics of Story, Berlin/New York 1990, sowie zum Zusammenhang zwischen Familie und narrativen Modellen in Hinsicht auf »eine Geschichte der modernen Männlichkeit« Walter Erhart: Familienmänner. Über den literarischen Ursprung moderner Männlichkeit, München 2001, hier S. 61.

9 Tobin: Time and the Novel (wie Anm. 7), S. IX u. 7.

10 "The novel offers, then, not a mimesis of undeliberated, organic life-in-time, but a homologue that enacts a privileged conceptualization of human life as purposeful and therefore imbued with meaning." Ebd., S. 5.

11 Ebd., S. 12.

12 Vgl. Sigrid Weigel: Genea-Logik. Generation, Tradition und Evolution zwischen Kulturund Naturwissenschaften, München 2006. 
oder des Verfalls - zu erzählen. ${ }^{13}$ Die mit solchen Erzählverfahren verbundene heterosexuelle Organisation der marriage und reproduction plots wird in den Romanen teils im heteronormativen Sinne unkommentiert vollzogen und so affirmiert, ${ }^{14}$ teils jedoch auf ihre Ein- und Ausschlussverfahren hin reflektiert - auch in dieser Hinsicht kann eine Untersuchung der Verweigerungsgeschichten, wie sie im Folgenden in Form von >Vatermordk- oder Junggesellennarrativen erfolgen wird, aufschlussreich sein.

Zudem richten sich solche Romane auch auf den Tod, der in ihnen keine Sprach- oder "Erkenntnisgrenze ${ }^{15}$ markiert, sondern im Gegenteil in die Sinnstiftung integriert wird; darauf hat Walter Benjamin eindringlich hingewiesen. Benjamin verortet die Bedeutung des Romans für seine Leser/ innen nämlich nicht in der lehrreichen Darbietung eines fremden Schicksals, sondern in dem Gewinn, den die Romanleser/innen aus dem Tod der Romanfigur ziehen. Denn da der Lebenssinn der Figur sich »erst von ihrem Tode her erschließt«, muss der Leser "im voraus gewiß sein, daß er ihren Tod miterlebt«, damit »dieses fremde Schicksal kraft der Flamme, von der es verzehrt wird, die Wärme an uns abgibt, die wir aus unserem eigenen nie gewinnen. Das was den Leser zum Roman zieht, ist die Hoffnung, sein fröstelndes Leben an einem Tod, von dem er liest, zu wärmen. ${ }^{16}$ Mit diesem drastischen Bild eines Sich-Wärmens am Tod anderer, das möglicherweise das eigene Überleben sichert, kritisiert Benjamin den "Mythos des Lebens«, wie er sich nicht nur in Wissenschaften und Philosophie, sondern auch in literarischen Schreibweisen und Romanformen des 19. Jahrhunderts, die das Leben beschwören, manifestiert - gut zu beobachten beispielsweise in Émile Zolas Rougon-Macquart-Romanzyklus, der gleich betrachtet werden soll.

Wie weitreichend solche vermeintlich lebensnahen, ja `organischen Romanauffassungen waren und sind, zeigt sich in Roland Barthes' Essay Literatur und Diskontinuität (1962), in dem er den umstrittenen Roman Mobile von Michel Butor (1961) gegen »die traditionelle Idee vom Buch« - der Mobile nicht entspricht - verteidigt:

13 Vgl. dazu auch Jobst Welge: Genealogical Fiction. Cultural Periphery and Historical Change in the Modern Novel, Baltimore 2015.

14 Vgl. z.B. Judith Roof: Come As You Are. Sexuality and Narrative, New York 1996.

15 Thomas Macho: Tod und Trauer im kulturwissenschaftlichen Vergleich, in:Jan Assmann: Der Tod als Thema der Kulturtheorie. Todesbilder und Totenriten im Alten Ägypten, m.e. Beitrag v. Thomas Macho, Frankfurt a.M. 2000, S. 89-120, hier S. 91.

16 Walter Benjamin: Der Erzähler. Betrachtungen zum Werk Nikolai Lesskows, in: Ders.: Gesammelte Schriften, hg. von Rolf Tiedemann und Hermann Schweppenhäuser, Bd. II.2, Frankfurt a.M. 1991, S. 438-465, hier S. $456 f$. 
Das (traditionelle) Buch ist ein Objekt, das verknüpft, entwickelt, fliest, sich fortspinnt, das heißt, das den größten Horror vor dem Leeren hat. [...] Auf der einen Seite das `Gesponnene` der lebendigen organischen Substanzen, die bezaubernde Unvorhersehbarkeit der spontanen Verknüpfungen, auf der anderen Seite das Sterile, Unharmonische mechanischer Konstruktionen, kalter knirschender Maschinen [so die gegen Butor erhobenen Vorwürfe] [...]. Denn hinter dieser Verdammung des Diskontinuierlichen verbirgt sich natürlich der Mythos vom Leben. ${ }^{17}$

Barthes erläutert dann das - von Butor und ihm kritisierte - Programm einer Literatur, die im Sinne eines solchen Mythos des Lebens verfasst werde, indem »die Wörter innerhalb der großen, Erzählen genannten Kategorie der Kontinuität fließen", und deren Ziel »ein Fluß von Wörtern im Dienst eines Ereignisses oder einer Idee [sei], die ihren Weg, auf die Auflösung oder die Schlußfolgerung zu, geht. ${ }^{18}$ Barthes' Kritik am "Mythos des Lebens" entspricht der an den "Mythologien« des modernen Alltags im Allgemeinen: Immerzu verschleiern Mythen durch Naturalisierungen das Geworden- und vor allem Gemachtsein von etwas, hier des Erzählens. In Barthes' analytischer Perspektive wird Literatur so als eine Bewältigungsstrategie von Kontingenz lesbar - eine Funktion, die im hier zu diskutierenden Zusammenhang von Leben, Vererbung und der Literatur des 19. Jahrhunderts insbesondere Zolas Rougon-Macquart-Romanzyklus kennzeichnet. Dieser zwanzigbändige Zyklus über fünf Generationen der Familie RougonMacquart vertritt sowohl das Prinzip des genealogischen Imperativs als auch den damit zusammenhängenden Mythos des Lebens: »la vie« steht mehrfach als letztes Wort am Romanende. Der im gesamten Rougon-Macquart-Zyklus immer wieder hervortretende unbändige Vitalismus stört allerdings die programmatische Erzählung eines konsequenten Verfalls und sorgt für eine Relativierung des Leitkonzepts der dégénérescence. ${ }^{19}$ Zudem ist der beschworene Lebensstrom in erzähltechnischer Hinsicht ein Problem, erfordert er doch eine souveräne Auktorialität, die aus einem ungreifbaren Kontinuum - »la vie aux courants infinis«, »indifférente aux hypothèses ${ }^{20}$ - einzelne Segmente isoliert und so überhaupt erkennbar und erzählbar macht. Damit aber werden diese Segmente zugleich in ihrer Kontingenz gezeigt, was die Auk-

17 Roland Barthes: Literatur und Diskontinuität. Über »Mobile« von Michel Butor [1962], in: Ders.: Literatur oder Geschichte, Frankfurt a.M. 1969, S. 85-101, hier S. $87 f$.

18 Ebd., S. 88.

19 Vgl. Marc Föcking: Pathologia litteralis. Erzählte Wissenschaft und wissenschaftliches Erzählen im französischen 19. Jahrhundert, Tübingen 2002, S. 343f.

20 Émile Zola: Les Rougon-Macquart. Histoire naturelle et sociale d'une famille sous le second Empire, hg. von Henri Mitterand, Paris 1960ff., Bd. 5, S. 1219. 
torialität wiederum entscheidend schwächt. ${ }^{21}$ Der letzte Roman des Zyklus, Le Docteur Pascal (1893), arbeitet an diesem Problem, indem er eine solche Autorposition dem Protagonisten Docteur Pascal aufbürdet, der als ein 'Meta-Autor`sämtlicher Romane sowie des Lebens selbst fungiert: Er hat die Lebens- und Krankengeschichten aller Familienmitglieder gesammelt und systematisiert sie gemäß verschiedener Vererbungsgesetze. Allerdings wird diese auktoriale Souveränität im selben Roman dem Tod überantwortet, wenn Docteur Pascal, bis zuletzt schreibend, stirbt. ${ }^{22}$ Das schreibende Subjekt - ob nun Docteur Pascal oder Émile Zola - vermag nichts anderes, als die Kontingenz seiner eigenen Existenz in eine Logik der Abfolge, eine Genealogie der Familienordnung bzw. eine literarische Tradition des Erzählens einzuschreiben. Dieser Akt der Souveränität, sich seine literarische Tradition zu erschreiben, muss sich jedoch letztlich der Kontingenz überantworten, dem unabsehbar, aber unausweichlich eintretenden Tod als dem Ende der individuellen Existenz und Abgrund zwischen den Generationen. Die Technik der Verkettung zur Reduktion von Kontingenz tritt in Le Docteur Pascal zum einen in Gestalt von Pascals Arbeit mit den zeitgenössischen Vererbungstheorien eines Darwin, Haeckel, Galton, Weismann und Lucas auf, wenn er die »enchaînements [...] de la descendance« ${ }^{23}$ d.h. die genealogisch-genetische Verkettung der Familie Rougon-Macquart erforscht. Zum anderen findet sich die Technik der Verkettung in der Romanserie selbst: Deren zwanzig Bände sind als experimentelle Reihe zu begreifen, die bei allen digredierenden Zügen auf synchroner Ebene die Art ihrer Verbindung auf diachroner Ebene erweisen soll. ${ }^{24}$ So spricht Zola in der Préface zur gesamten Serie von der auf den ersten Blick zu konstatierenden Unähnlichkeit der auftretenden Figuren, deren innere liaison durch eine Analyse

21 Für »das Erzählsubjekt des Romanciers« in Zolas La Fortune des Rougon beschreibt Marc Föcking »die Konsequenzen einer Auktorialität aus epistemologischer Schwäche: Die Segmentierungen, die Isolierungen von Objekten aus diesem Lebensstrom sind einesteils unumgänglich, um zu Erkenntnis- wie Erzählobjekten zu kommen, aber sie sind andererseits stets kontingent und arbiträr«. Föcking: Pathologia litteralis (wie Anm. 19), S. 325.

22 Vgl. dazu Ulrike Vedder: Das Testament als literarisches Dispositiv. Kulturelle Praktiken des Erbes in der Literatur des 19. Jahrhunderts, München 2011, S. 381.

23 Zola: Les Rougon-Macquart (wie Anm. 20), Bd. 5, S. 1739.

24 In dieser Hinsicht spricht Gumbrecht von zwei zentralen Problemen, "mit denen Zola [...] bei der Planung seines Werkes konfrontiert war: nämlich die Interferenzen zwischen verschiedenen Gesetzen diachronischen Ablaufs aus Bereichen qualitativ verschiedener Determinanten und die Zuordnung von synchronischer und diachronischer Perspektive.« Hans Ulrich Gumbrecht: Zola im historischen Kontext. Für eine neue Lektüre des Rougon-Macquart-Zyklus, München 1978, S. 41. 
im Paradigma der Vererbung sichtbar zu machen sei, um jenen Faden zu finden, "qui conduit mathématiquement d'un homme à un autre homme. ${ }^{25}$ Damit nutzt Zola das Konzept einer gesetzmäßigen Vererbung nicht nur als Referenz auf ein prestigeträchtiges wissenschaftliches Modell oder als Erkenntnisinstrument, sondern auch als außerordentlich produktiven Romangenerator. So veröffentlicht Zola mit seinem achten Roman Une Page d'amour (1878) einen Stammbaum der Familie Rougon-Macquart, »'arbre généalogique«, den er als "ma force et mon régulateur bezeichnet $^{26}$ - als eine wissenschaftlich-erzählerische Technologie mithin, die neben der Funktion eines Darstellens von Wissen auch die eines Generierens von Wissen und nicht zuletzt eines Generierens von Erzählungen hat, das dem genealogischen Imperativ folgt. Zolas ausufernde epische Schilderungen in zwanzig Bänden sichern ihre Überschaubarkeit in vielfacher Weise: mit Hilfe ihrer Paratexte - u.a. des Stammbaums -, mit Hilfe ihrer Kasuistik, mit Hilfe ihrer engmaschigen hereditär-intergenerationellen Verkettungen sowie mit Hilfe ihrer Zusammenfassung im abschließenden Roman Le Docteur Pascal. Solche Maßnahmen zur Reduktion von Kontingenz und zur Herstellung von Übersichtlichkeit stellen sicherlich eine Voraussetzung dar, um Zolas Literatur wiederum für die Wissenschaft nutzbar machen. So verwendet der Psychiater Robert Sommer in seiner Studie Familienforschung und Vererbungslehre (1907) Zolas fiktionale Daten der Familie Rougon-Macquart als Material für seine genealogisch orientierten Untersuchungen. ${ }^{27}$

\section{Verweigerung der Vererbung: Möglichkeiten des antigenealogischen Romans}

Wenn es nun, nach dem Paradigma des genealogischen Romans, um die Negation des genealogischen Imperativs, d.h. um die erzählerisch so produktive Verweigerung von Fortpflanzung, Vererbung, Geschlechtlichkeit, intergenerationeller Verknüpfung geht, so ist damit an zentraler Stelle die Infragestellung einer familialen, hereditären und literarischen Vaterposition angesprochen: "So wie die narrative Genealogie im 19. Jahrhundert den männlichen Geschlechtscharakter unter Beweis stellt, so ist dieser durch

25 Zola: Les Rougon-Macquart (wie Anm. 20), Bd. 1, S. 3.

26 Ebd., Bd. 2, S. 799.

27 Vgl. Robert Sommer: Familienforschung und Vererbungslehre, Leipzig 1907, S. 206-210. 
den Abbruch der Genealogie in Frage gestellt. ${ }^{28}$ Dabei gilt es nicht nur, den Vater abzuschaffen, um schreiben zu können und jener übermächtigen Figur zu entkommen, die zwar erzählträchtige Konflikte erzeugt, aber keine Anerkennung des Sohnes und seiner Schreibkunst leistet. Sondern es gilt darüber hinaus - als eine Variante des antigenealogischen Romans -, sich des toten Vaters zu entledigen. Ein solcher Versuch findet sich in Form zweier starker Szenen in zwei Romanen, die nicht zufällig als Meilensteine der Modernisierung des Romans gelten: in Herman Melvilles Pierre or, The Ambiguities und in Rainer Maria Rilkes Die Aufzeichnungen des Malte Laurids Brigge. Beide Romane schildern den Versuch der Söhne - deren Namen im Titel stehen und die sich im Roman als Autoren etablieren wollen -, auf antigenealogische Weise "ich" zu sagen, und beide verfolgen das in einer experimentellen Schreibweise, die die gängigen Romanformen explorativ herausfordert. Beide Szenen der Beseitigung eines bereits toten Vaters seien nun genauer auf ihre antigenealogische Reichweite hin betrachtet, bevor dann ein weiteres Modell dezidiert antigenealogischen Schreibens erörtert werden soll: der Junggesellenroman.

In Herman Melvilles Roman Pierre or, The Ambiguities (1852) ist die Familie unausweichlich, auch wenn sie zunächst nur aus einer Mutter und deren einzigem Sohn besteht, während der geliebte Mann bzw. Vater seit langem tot ist. Doch diese Zwei-Personen-Familie, die Glendinnings, vervielfältigt sich selbst von Beginn an, noch bevor Pierres Halbschwester Isabel auftaucht: Mutter und Sohn sprechen sich voller Liebe als Bruder und Schwester an, wodurch eine inzestuöse Dimension des Familialen ins Spiel kommt; die toten Vorfahren sind geradezu überpräsent, wodurch die genealogische Dimension des Familialen gegenwärtig ist. Zwar ist das Geschehen in Neuengland angesiedelt, mithin in der 'Neuen Welt , in der, so die alteuropäische Vorstellung, das Leben in einer vergangenheitslosen Gegenwart stattfinde. Doch gegenläufig dazu ist Pierre am Beginn des Romans mit seiner Herkunft und seinen Vorfahren völlig identifiziert: »daß auf jene Hügel schon seine wackeren Väter geblickt hatten, daß durch jene Wälder [...] schon so manche seiner Ahnfrauen als junges Mädchen fröhlich dahingeschlendert war, [...] und selbst der Horizont erschien ihm wie ein Gedächtnisring. « ${ }^{29}$ Der Bruch in diesem Kontinuum setzt mit dem Auftauchen Isabels ein, einer bis dahin unentdeckten illegitimen Tochter des verehrten verstorbenen

28 Erhart: Familienmänner (wie Anm. 8), S. 111.

29 Herman Melville: Pierre oder Die Doppeldeutigkeiten, übers. von Christa Schuenke, München/Wien 2002, S. 20; vgl. zum Folgenden auch Vedder: Das Testament als literarisches Dispositiv (wie Anm. 22), S. 325-329. 
Vaters. Um die Mutter vor diesem Wissen zu bewahren und die Halbschwester aus Armut und Einsamkeit zu retten, verlässt Pierre das idyllische Familiengut, gibt Isabel als seine Ehefrau aus und geht mit ihr sowie mit seiner zur Cousine erklärten früheren Braut Lucy nach New York, wo er Schriftsteller werden will. Die entsetzte Mutter enterbt und verstößt ihn, bevor sie vor Kummer stirbt; am Romanschluss steht der gemeinsame Tod der drei jungen Leute und damit das Ende des gesamten Geschlechts der Glendinnings.

In dieser Geschichte spielt immer wieder ein Porträtgemälde des toten Vaters eine Rolle, auf dem er, "smiling and ambiguous «, ${ }^{30}$ besonders lebendig und anziehend erscheint. Pierre hat dieses geliebte Bild heimlich in seinen Besitz gebracht, während die Mutter ein konventionelles repräsentatives Gemälde ihres Mannes vorzieht. Das slebendige< Bild des toten Vaters wird nach der Begegnung mit Isabel einerseits zum Beweisstück ihrer Ähnlichkeit mit dem Vater - andere Belege der Vaterschaft gibt es nicht -, andererseits zum emotionalen Fluchtpunkt des verstoßenen Pierre, der dem Porträt nun allerdings voller Hass begegnet. Dafür gibt der Erzähler eine ebenso diffuse wie weitreichende Erklärung, in der dem Bild »intelligence and vitality« zugeschrieben werden, ja mehr noch: Zeugungskraft und Vaterschaft:

Es war, als lauerte in diesem Bilde irgendeine geheimnisvolle Kenntnis und Lebendigkeit [intelligence and vitality], denn da sich Pierre in seiner eigenen Erinnerung an seinen Vater auf keinen bestimmten Zug besinnen konnte, der etwa auf Isabel übergegangen war, sondern in dem Porträt nur eine ungefähre Ähnlichkeit erkannte, darum kam es ihm so vor, als wär der wahre Vater Isabels jenes gemalte Bildnis selbst und nicht der leibliche Vorfahr [...]; denn [...] Isabel [hatte] einen ganz bestimmten Zug geerbt, der nur in diesem Bildnis festzustellen war, sonst nirgendwo. ${ }^{31}$

Wenn Pierre das Porträt dann verbrennt, so kann dies als der Versuch verstanden werden, mit dem Vaterbild auch den toten Vater gänzlich zu zerstören, um so dessen fatale Lebendigkeit, die genealogische Bindung und die ambiguities zu beseitigen - ein Versuch, sich von jenen omnipräsenten Modi der »Verwandtschaft, Wechselbeziehung und Übertragung « ${ }^{32}$ zu befreien, die den Roman und all seine Figuren und Geschehnisse bestimmen.

Mit der emphatischen Lossagung von Familie, Genealogie, Vererbung und Vergangenheit will Pierre sich selbst als 'Enterbten in die totale Gegenwart

\footnotetext{
30 Herman Melville: Pierre or, The Ambiguities, New York 1963, S. 274.

31 Melville: Pierre oder Die Doppeldeutigkeiten (wie Anm. 29), S. 342f.

32 Ebd., S. 342.
} 
und in die Freiheit seines Selbst entlassen: "cast-out Pierre hath no paternity, and no past, [...] twice-disinherited Pierre stands untrammelledly his ever-present self! - free to do his own self-will and present «. ${ }^{33}$

Doch angesichts der skizzierten Überlagerungen und Verstrickungen kann dies nicht das letzte Wort sein, denn bis in die Bilder, die Figuren, die Namen, die Familienstrukturen, die Handlungen und die sprachliche Rhetorik des Romans hinein geht es permanent um Reproduktion. In Pierre ist das Prinzip der Wiederholung mit dem der Deszendenz unausweichlich verkettet: sei es durch den Drang der Protagonisten, sich in fast jeder Äußerung zu wiederholen und Ausrufe immer gleich mehrfach auszustoßen; sei es durch die Eintönigkeit, mit der beispielsweise, wenn von Pierres Großvater namens Pierre erzählt wird, immer wieder dessen Name "grand old Pierre" wiederholt wird; sei es durch die geringen Variationen der immer gleichen Wortfamilie, wie etwa »descendence ${ }^{34}$ sei es durch den repetitiven Erzähler, der seine Wiederholungen geradezu ausstellt in Wendungen wie "it has been said", "we have before claimed", »the same grandfather several times herein-before mentioned ${ }^{35}{ }^{35}$ sei es durch Kapitelüberschriften, wie etwa "More light, and the gloom of that light; more gloom, and the light of that gloom«. Wörter und Formulierungen wiederholen sich, stammen voneinander ab oder spiegeln sich ineinander, ebenso wie die Figuren dies tun, die simmer schon untereinander verwandt sind.

Zur exzessiv reproduktiven Struktur trägt zudem die enorme intertextuelle Anspielungsbreite - mit Verweisen u.a. auf Dante, Shakespeare, Byron, Wordsworth, Dickens, Poe sowie u.a. auf den Schauerroman, den Bildungsroman, den psychologischen Roman ${ }^{36}$ - ebenso bei wie der Protagonist Pierre, der, als Romanfigur, an einem autobiographischen Roman über einen Schriftsteller schreibt. Dieser Schriftsteller in Pierres Roman

33 Melville: Pierre or, The Ambiguities (wie Anm. 30), S. 277.

34 Cindy Weinstein zeigt die vielfache Wiederholung solcher Varianten auf wenigen Seiten; allein in Kap. I.3 tauchen mehrfach auf: descended, endless descendedness, blooddescent, far-descended, descending, double-revolutionary descent; Cindy Weinstein: We are Family. Melville's »Pierre«, in: Leviathan. A Journal of Melville Studies 7/1 (2005), S. 19-40.

35 Melville: Pierre or, The Ambiguities (wie Anm. 30), S. 8, S. 13 u. 15.

36 Helmbrecht Breinig bezeichnet Pierre als »Darstellung des komplexen Wechselbezugs von problematischer Genealogie und ebenso problematischer Intertextualität, wobei die biologische und die textuelle Abstammungslinie sich allenfalls bis zu jener undurchschaubaren, stummen und vielleicht abwesenden/leeren Instanz verfolgen lassen, die die Menschheit als `Gott bezeichnet." Helmbrecht Breinig: Satire und Roman. Studien zur Theorie des Genrekonflikts und zur satirischen Erzählliteratur der USA von Brackenridge bis Vonnegut, Tübingen 1984, S. 146. 
trägt ausgerechnet den Namen Vivia und erscheint damit, versteht man ,Vivia als Ableitung von 'Leben bzw. >Verlebendigung (vivification), wie ein Zerrbild von sPierrer (petrification). Folglich wird der Roman um Vivia das Leben Pierres nicht sichern können, im Gegenteil: Als Pierre sich am Ende erschießt, lädt er die Pistole nicht nur mit einer Kugel, sondern auch mit einem Brief seines Verlegers, der die Rückzahlung von Vorschüssen für nie geschriebene Romane fordert.

Pierres antigenealogischer Wunsch nach Subjektivität, Befreiung und Gegenwart (»free to do his own self-will and present») wird durch das Verbrennen des väterlichen Porträts befördert. Doch dem steht die im Roman exzessiv inszenierte und letztlich tödliche Übermacht der Reproduktion - im biologischen und ästhetischen Sinne - entgegen. Folglich inszeniert Melvilles Roman auch das Ungenügen an den gängigen Romanformen des 19. Jahrhunderts, indem er diese so konsequent zitiert, parodiert und rekombiniert, dass Pierre or, The Ambiguities als ein unlesbarer, sverrückter Text von der zeitgenössischen Rezeption verworfen wurde.

Einige Jahrzehnte später haben sich »die Subjektivität der Verweigerung« und »der allen bourgeoisen Werten entgegenstehende Protagonist der Lebensuntüchtigkeit, [...] Verkörperungen lebens-vergewaltigender oder lebens-desinteressierter Ästheten « in der Literatur etabliert ${ }^{37}$ - man denke exemplarisch an Jean Floressas des Esseintes in Joris-Karl Huysmans' Roman $\grave{A}$ Rebours (1884) oder an Oscar Wildes Protagonisten Dorian Gray (1891), die ebenfalls explizit als die letzten ihres jeweiligen Geschlechts auftreten. In Rainer Maria Rilkes Aufzeichnungen des Malte Laurids Brigge (1910) verdankt sich die Kohäsion des Romans nur mehr der Konstruktion eines subjektiven Bewusstseins, das qua Assoziation und Aufzeichnung die wahrgenommene Welt und die eigene Positionierung darin zu fassen sucht. Darüber hinaus ist es auch die Radikalität einer Szene mit dem toten Vater, die den schreibenden Protagonisten in einem offensiv antigenealogisch gedachten «Leben verorten soll.

Gemeint ist jene Szene, in der Maltes Vater nach seinem Tod den Herzstich erhält - mithin eine zunächst anachronistisch anmutende Szene, ist doch die Angst vor dem Scheintod, die die Technik des Herzstichs motiviert, vor allem eine des 18. Jahrhunderts. ${ }^{38}$ Doch taucht sie zu Beginn des 20. Jahrhunderts, nach dem Ende der großen wissenschaftlichen und populären

37 Margret Eifler: Die subjektivistische Romanform seit ihren Anfängen in der Frühromantik, Tübingen 1985, S. 47.

38 Vgl. etwa Martina Kessel: Die Angst vor dem Scheintod im 18. Jahrhundert. Körper und Seele zwischen Religion, Magie und Wissenschaft, in: Thomas Schlich/Claudia Wiese- 
Scheintoddebatten, als eine technische und zugleich mythisierte Geste vermehrt wieder auf. ${ }^{39}$ Bei Rilke nun markiert die >Tötung` des toten Vaters qua Herzstich zugleich die antigenealogische Selbstbegründung des Ich, das nicht zufällig, ebenso wie Pierre, sich als Dichter in einer Großstadt zu etablieren sucht. Malte erinnert sich:

Wer hätte zum Beispiel an diesen Widerstand gedacht. [...] das rasch angesetzte Instrument drang nicht ein. Ich hatte das Gefühl, als wäre plötzlich alle Zeit fort aus dem Zimmer. Wir befanden uns wie in einem Bilde. Aber dann stürzte die Zeit nach mit einem kleinen, gleitenden Geräusch [...]. Auf einmal klopfte es irgendwo. Ich hatte es noch nie so klopfen hören: Ein warmes, verschlossenes, doppeltes Klopfen. Mein Gehör gab es weiter, und ich sah zugleich, daß der Arzt auf Grund gestoßen war. [...] So, so, dachte ich, nun ist es also durch. [...] nun war [er] tot, und nicht er allein. Nun war das Herz durchbohrt, unser Herz, das Herz unseres Geschlechts. Nun war es vorbei. [...] »Heute Brigge und nimmermehr«, sagte etwas in mir. An mein Herz dachte ich nicht. Und als es mir später einfiel, wußte ich zum erstenmal ganz gewiß, daß es hierfür nicht in Betracht kam. Es war ein einzelnes Herz. Es war schon dabei, von Anfang anzufangen. ${ }^{40}$

Der Herzstich garantiert hier nicht nur die endgültige Sicherung des väterlichen Todes, sondern vor allem die endgültige Trennung des Ich vom Vater und vom gesamten Geschlecht der Brigges. Das Herz des Ich, »ein einzelnes Herz«, ist also nicht länger ein genealogisches Organ, das im Tode, durch den Herzstich abgesichert, das Leben auf künftige Generationen überträgt. Vielmehr ist das schreibende Ich mit dem vollendeten Herzstich, d.h. mit der Gewissheit, dass der Vater so tot ist, dass er nicht wiederkehren wird, seiner selbst »zum erstenmal ganz gewiß«. Durch die Lossagung von Vater, Familie, Genealogie gewinnt das Ich den autonomen Status als selbstgeschaffenes »einzelnes Herz«, »dabei, von Anfang anzufangen«.

Das Schaffen eines solchen herkunftslosen Anfangs bildet auch das antigenealogische Ideal der Junggesellenmaschinen um 1900, um das es nun abschließend gehen soll. Am Ende des 19. Jahrhunderts ist der Junggeselle

mann (Hg.): Hirntod. Zur Kulturgeschichte der Todesfeststellung, Frankfurt a.M. 2001, S. 133-166.

39 Der Herzstich ist von Ärzten und Literaten wie etwa von Arthur Schnitzler (gest. 1931) selbst testamentarisch verfügt worden. In Kafkas Tagebuch seiner Italienreise mit Max Brod heißt es im Eintrag vom 1.9.1911: „Gespräch über Scheintod und Herzstich an einem Kaffeehaustischchen auf dem [Mailänder] Domplatz. Mahler hat auch den Herzstich verlangt.« Franz Kafka: Tagebücher, hg. von Hans-Gerd Koch u.a., Frankfurt a.M. 1990, S. 966. Nicht zuletzt hat auch Rainer Maria Rilke selbst beim Tod seines Vaters im Jahr 1906, so hatten beide es verabredet, den Herzstich angeordnet.

40 Rainer Maria Rilke: Die Aufzeichnungen des Malte Laurids Brigge, in: Sämtliche Werke, hg. von Ernst Zinn, Bd. 6, Frankfurt a.M 1987, S. 707-946, hier S. 854f. 
in der Literatur als eine Figur prominent, ${ }^{41}$ die eine lange Generationenkette abbricht und mit ihrer genealogischen Unfruchtbarkeit das Ende der Zukunft verkörpert. So wird der Junggeselle zum Angriff auf die Macht der Vererbung, der Prokreation, des Naturalismus und des sogenannten Lebens stilisiert, wie sie sich am Ende des 19. Jahrhunderts etabliert hat. Einen solchen Angriff führen die künstlerischen Avantgarden des frühen 20. Jahrhunderts mit dem Produktionsmodell der »Junggesellenmaschine ${ }^{42}{ }^{4}$ d.h. mit erotisch aufgeladenen Maschinen und Apparaten, die Gegenmodelle zur natürlichen Reproduktion darstellen. Sie dienen der Erregung und Bewegung, der Sexualiät und dem Energiekreislauf und sind als sich selbst antreibende Mechanismen konzipiert, die, in keine Teleologie eingespannt, sich den üblichen Restriktionen der Ökonomie sowie den Gesetzen der thermodynamischen Physik entziehen können sollen.

Schon in Marcel Duchamps Kommentar von 1913 zu La Mariée mise à nue par ses célibataires, même (Die Braut, von ihren Junggesellen nackt entblöst, sogar) (1912-1923), auch genannt Gro/ses Glas, findet sich die Benennung "Junggesellenmaschine « ${ }^{43}$ für den unteren Teil seines Kunstwerks. Jenen Begriff verwendet Michel Carrouges in seinem Essay Die funggesellenmaschinen (1954) zur Bezeichnung von literarischen und künstlerischen Werken, deren Struktur und Funktionsprinzipien denen des Grofsen Glases ähneln: Werke etwa von Edgar Allen Poe, Alfred Jarry, Raymond Roussel, Franz Kafka, Giorgio de Chirico, in denen ebenso phantastische wie technisch ausgereifte Maschinen imaginiert werden, die Mechanik und Erotik vereinen und von jeglicher Zeugungsfunktion - und damit von der Idee familialer Reproduktion, Deszendenz und Genealogie - entkoppelt sind.

Dabei geht es nicht nur um die Verweigerung der Prokreation und der an sie gekoppelten Bedeutsamkeit. Vielmehr besteht das Ziel in einer Neuausrichtung der Kreativität im Zeichen einer Abwesenheit von Nachkommen, wo-

41 Vgl. etwa Nathalie Prince: Les Célibataires du fantastique. Essai sur le personnage célibataire dans la littérature fantastique de la fin du XIXème siècle, Paris 2002; Katherine V. Snyder: Bachelors, Manhood, and the Novel 1850-1925, Cambridge 1999; Annette Runte (Hg.): Literarische Junggesellen-Maschinen und die Ästhetik der Neutralisierung, Würzburg 2011; Ulrike Vedder: Reproduktion in Gefahr. Männliche Junggesellen in Literatur und Wissenschaften des 19. Jahrhunderts, in: Andreas Heilmann u.a. (Hg.): Männlichkeit und Reproduktion. Zum gesellschaftlichen Ort historischer und aktueller Männlichkeitsproduktionen, Wiesbaden 2015, S. 43-58.

42 Vgl. Hans Ulrich Reck/Harald Szeemann (Hg.): Junggesellenmaschinen, Wien/New York 1999.

43 Vgl. Jean Clair: Vier Daten, in: Reck/Szeemann (Hg.): Junggesellenmaschinen (wie Anm. 42), S. 13. 
durch die Idee menschlicher Verwandtschaft qua natürlicher Fortpflanzung und damit auch Konzepte biologischer Vererbung und bürgerlicher Familie mit all ihren Implikationen verabschiedet werden sollen: zugunsten einer "Totem-Verwandtschaft mit der Maschine «, ${ }^{44}$ d.h. einer Mann-MaschineKonstellation, die ihren mythologischen Charakter ausstellt. In vielen dieser zölibatären Entwürfe wird allerdings der weibliche Körper demontiert oder fragmentiert und als Material genutzt: Wie dies schon Herman Melvilles Erzählung The Paradise of Bachelors and the Tartarus of Maids (1855) imaginiert - zwar nicht als Junggesellenmaschine im Sinne der späteren künstlerischen Avantgarden, jedoch deren Funktionsweisen präfigurierend. Die künstlerischen Junggesellenmaschinen des frühen 20. Jahrhunderts sind also darauf aus, einerseits sexuelles Begehren ohne Frauen bzw. Weiblichkeit als rreine Lust $\mathrm{zu}$ verwirklichen und andererseits eine »ewig, mühelos funktionierende Maschine« ohne Abnutzung, mithin ein perpetuum mobile als reine Kreation $\mathrm{zu}$ schaffen. ${ }^{45}$

Dass in dieser von bürgerlicher Liebe und Generativität, von Weiblichkeit und Vitalität sgereinigten Junggesellenästhetik Destruktivität und Todessehnsucht eine zentrale Rolle spielen, analysieren beispielsweise die Werke von Franz Kafka. Wenn Kafkas Literatur die Figur des Junggesellen und diverse Spielarten der Junggesellenästhetik und -maschinerie einsetzt, so handelt es sich, wie Timothy Attanucci betont, "nicht nur um eine literarische Verarbeitung persönlicher Lebensentscheidungen, sondern um die Frage der Organisation biologischer, ökonomischer, sozialer sowie kultureller Produktion und Reproduktion. " ${ }^{46}$ Vor allem aber verbindet Kafkas Literatur eine dezidiert antigenealogische Thematik und Ästhetik einerseits mit dem Begehren nach einem Zusammenhang jenseits von Familie, bei gleichzeitiger Trauer um das Verfehlen dieses Begehrens, ${ }^{47}$ und andererseits mit einer Kritik am literarisch kreierten Mythos des Lebens - die Benjamin und

44 Michel Carrouges: Gebrauchsanweisung, in: Reck/Szeemann (Hg.): Junggesellenmaschinen (wie Anm. 42), S. 74-105, hier S. 103.

45 »Indem die Junggesellenmaschine den Gesetzen, die unser physikalisches und physiologisches Universum bestimmen, entgeht, verschafft sich ihre Junggesellentätigkeit Zutritt zu den Gefilden reiner Kreation (’Kunst‘) und reiner Lust (Erotik). "Jean Clair: Zwei Interpretationen, in: Reck/Szeemann (Hg.): Junggesellenmaschinen (wie Anm. 42), S. 16-18, hier S. 18.

46 Timothy J. Attanucci: Kafka: Junggeselle - Maschine. Überlegungen zu einer historischen Kopplung, in: Runte (Hg.): Literarische Junggesellen-Maschinen` (wie Anm. 41), S. 171.

47 Vgl. beispielsweise: „Ohne Vorfahren, ohne Ehe, ohne Nachkommen, mit wilder Vorfahrens-, Ehe- und Nachkommenslust. Alle reichen mir die Hand: Vorfahren, Ehe und Nachkommen, aber zu fern für mich.« Franz Kafka: Tagebücher 1910-1923, hg. von Max Brod, Frankfurt a.M. 1967, S. 402, 22.1.1922. 
Barthes mit ihm teilen -, wenn er seine Texte als Todeserzählungen kennzeichnet, an denen sich kein Leser wärmen kann: »Ich selbst aber kann nicht weiterleben, da ich ja nicht gelebt habe, ich bin Lehm geblieben, den Funken habe ich nicht zum Feuer gemacht, sondern nur zur Illuminierung meines Leichnams benutzt. ${ }^{48}$

48 Brief an Max Brod, 5.7.1922, Franz Kafka: Briefe 1902-1924, hg. von Max Brod, Frankfurt a.M. 1966, S. 385. 